\title{
Downregulation of TMPRSS4 Enhances Triple-Negative Breast Cancer Cell Radiosensitivity Through Cell Cycle and Cell Apoptosis Process Impairment
}

\author{
Ganiou Assani, ${ }^{1,2}$, Julien Segbo ${ }^{3}$, Xiaoyan Yu',2, Akadiri Yessoufou ${ }^{3}$, Yudi Xiong,2 \\ Fuxiang Zhou ${ }^{1,2}$, Yunfeng Zhou ${ }^{1,2 *}$
}

\begin{abstract}
Background: Radioresistance remains a challenge for cancer radiotherapy. The present study aims to investigate the role of TMPRSS4 in triple negative breast cancer (TNBC) cell radiosensitivity. Materials and Methods: After transfection of MDA-MD-468 triple negative breast cancer cells line by using the lentivirus vector, the effect of TMPRSS4 down-regulation on TNBC radiosensitivity was evaluated by using cloning assay and CCK-8 assay. The CCK-8 assay was also used for performing cell proliferation analysis. Western blot was carried out to detect the expression of certain proteins related to cell cycle pathways (cyclin D1), cell apoptosis pathways (Bax, Bcl2, and Caspase3), DNA damage and DNA damage repair (TRF2, Ku80, $\mathrm{rH} 2 \mathrm{AX}$ ). The cell cycle and cell apoptosis were also investigated using flow cytometer analysis. Results: TMPRSS4 expression was down-regulated in MDA-MB-468 cells which enhanced MDA-MB-468 cells radiosensitivity. TMPRSS4 silencing also improved IR induced cell proliferation ability reduction and promoted cell arrested at G2/M phase mediated by 6 Gy IR associated with cyclin D1 expression inhibition. Moreover, TMPRSS4 inhibition enhanced TNBC apoptosis induced by 6 Gy IR following by over-expression of (Bax, Caspase3) and down-regulation of $\mathrm{Bcl} 2$ as the pro-apoptotic and anti-apoptotic proteins, respectively. Otherwise, TMPRSS4 down-regulation increases DNA damage induced by 6 Gy IR and delays DNA damage repair respectively illustrated by downregulation of TRF2 and permanent increase of Ku80 and rH2AX expression at $1 \mathrm{~h}$ and $10 \mathrm{~h}$ postIR. Conclusion: Down-regulation of TMPRSS4 increases triple negative breast cancer cell radiosensitivity and the use of TMPRSS4 inhibitor can be encouraged for improving radiotherapy effectiveness in TNBC radioresistant patients.
\end{abstract}

Keywords: TMPRSS4 - Triple-negative breast cancer - Radiosensitivity - Cell cycle - Cell apoptosis

Asian Pac J Cancer Prev, 20 (12), 3679-3687

\section{Introduction}

Breast cancer is cancer frequently diagnosed in about $84 \%$ of countries in the world and the leading cause of cancer death in over $54 \%$ of world countries (Bray et al., 2018). In 2012, the number of the new cases occur was estimated at 1, 7 million and 520.000 deaths (Torre et al., 2012 and 2016; Lee et al., 2018,Chen et al., 2015) against 2, 1 million and 626.679 death in 2018 (Bray et al., 2018). Based on these data, breast cancer can be considered as an important public health issue and more research have to be encouraged for its preventive and curative treatment. Moreover, Triple negative breast cancer (TNBC), characterized by the lacks of expression of estrogen receptor (ER), progesterone receptor (PR) and human epidermal growth factor receptor2 (HER2) (Lukoseviciene et al., 2018), is the most aggressive among the subtype of breast cancer and represents about $15 \%$ of all case of breast cancer (Millikan et al., 2008; Yang et al., 2019; Abramson et al., 2015; Wen et al., 2019). However, due to its molecular characteristics, TNBC remains more difficult to treat with common breast cancer therapies and easily develop resistance to therapy including the radiotherapy (Foulkes et al., 2010; Agrawal et al., 2014; Lehmann et al., 2011; Augoff et al., 2012; Tian et al., 2017; Ilgun et al., 2016) which is useful because it contributes to more than $50 \%$ of cancer treatment (Wen et al., 2019; Kindts et al., 2017; Moran et al., 2015). For resolving the radiotherapy resistance problems of TNBC, it is therefore important to explore the new biomarkers that predict or improve the radiotherapy effectiveness.

A single-pass typeII membrane protein transmembrane protease serine4 (TMPRSS4) is a novel serine protease discovered at the cell surface. TMPRSS4 is highly expressed in several types of cancer cell lines (Chikaishi et al., 2016; Wu et al., 2014; Liang et al., 2013) especially

${ }^{1}$ Hubei Cancer Clinical Study Center, Hubei Key Laboratory of Tumor Biological Behaviors, ${ }^{2}$ Department of Radiation and Medical Oncology, Zhongnan Hospital, Wuhan University, Wuhan, Hubei, China, ${ }^{3}$ University of Abomey Calavi, BP 526, Cotonou, Benin.*For Correspondence: yfzhouwhu@163.com 
the TNBC cell line (Cheng et al., 2013; Li et al., 2017). TMPRSS4 is also implicated in cancer cell proliferation (Huang et al., 2014; Kim et al., 2010) and cancer radiotherapy ( $\mathrm{Li}$ et al., 2011). It has been shown that TMPRSS4 inhibition reduced cancer cell proliferation ability by promoting cell cycle arrested, cell apoptosis and enhanced radiotherapy effectiveness (Huang et al., 2011; Li et al., 2011; Fan et al., 2018; Lee et al., 2016; Jung et al., 2008). Moreover, cell cycle and cell apoptosis are two steps of cancer progression and are implicated in cancer treatment (Diaz-Moralli et al., 2013; Pistritto et al., 2016; Wong et al., 2011; Kadam et al., 2016) including radiotherapy (Assani et al., 2019; Pawlik et al., 2004; Meyn et al., 1997). However, the function of TMPRSS4 in cancer cell radiosensitivity especially the triple negative breast cancer remains unclear. In the present study, the stable TMPRSS4 down-regulating TNBC cell lines were used to explore the role of TMPRSS4 on triple negative breast cancer radiosensitivity in relationship with cell cycle and cell apoptosis. From our results, we demonstrated the negative correlation between TMPRSS4 expression and TNBC sensitivity to irradiation suggested that TMPRSS4 could be considered as a potential therapeutic target for enhancing breast cancer radiotherapy efficacy especially the triple negative breast cancer.

\section{Materials and Methods}

\section{Cell line and cell culture}

Human breast cancer cell (MDA-MB-468), one of the commonly used for TNBC studies, was obtained from the key laboratory of tumor biological behavior of Hubei province and was cultured in Dulbecco's modified eagle medium (DMEM, Hyclone, Logan, USA) supplemented with $10 \%$ fetal bovine serum (FBS, WiSant,Canada), 100 $\mathrm{U} / \mathrm{ml}$ penicillin and $100 \mu \mathrm{g} / \mathrm{mL}$ streptomycin under $37^{\circ} \mathrm{C}$ with $5 \% \mathrm{CO}_{2}$ humidified atmosphere.

\section{Vector and stably transfected cell lines development}

The lentivirus vectors PLKO.1-sh-TMPRSS4 versus PLKO.1-sh-NC (Bioeagle/Wuhan/China) were transfected in MDA-MB-468 cell lines. The stably transfected cell lines were selected at $5 \mu \mathrm{g} / \mathrm{mL}$ puromycin after two to three weeks post-transfection. The stable transfected cells lines developed were respectively named MDA-MB-468-shTMPRSS4 and MDA-MB-468-NC. The expression of TMPRSS4 was detected at mRNA and protein level by qPCR and western blot, respectively.

\section{Western blot analysis}

Western blot was implemented for the analysis of protein expression. The stable transfected MDA-MB-468 were washed twice with ice-cold phosphate-buffered saline (PBS) and were treated with radioimmuno-precipitation assay lysis buffer (\#P0013B, Beyotime biotechnology, China) containing 1XPMSF (\#ST506, Beyotime biotechnology, China) and phosphatase inhibitor tablets (\#4906837001, Roche, USA). For western blot assay, the same quantity of extracted protein was electrophoresed with $8-12,5 \%$ sodium dodecyl sulfate-polyacrylamide gel (SDS Page) and transfected to polyvinylidene difluoride
(PVDF) membrane with the transfer buffer following by the membrane blocking with $5 \%$ non-fat milk at room temperature for $2 \mathrm{~h}$. The membranes were incubated with the primary antibodies at $40 \mathrm{C}$ overnight and washed three times with Tris-buffered saline containing $0,1 \%$ of Tween-20 before its incubation with goat anti-rabbit or anti-mouse horseradish peroxidase-conjugated antibodies (1:10,000; respectively Lot 1387291024 and Lot 1357561012, Cell Signaling Technology) for $1 \mathrm{~h}$ at room temperature at least. The Tris-buffered saline containing $0,1 \%$ of Tween- 20 was used for washing the membranes three to five time( $10 \mathrm{~min}$ per washing) prior to each step. The primary antibodies used included: GAPDH (1:5,000,\#60004-1-Ig,Proteintech,China), TMPRSS4 (1:1,000 dilution,\#A4865, ABclonal,USA ), Cyclin D1 (1:1,000 dilution,\#60186-1-lg, Proteintech,China), Bax (1:1,000 dilution, \#50599-2-Ig, Proteintech,China ), Bcl2 (1:1,000 dilution,\#A16776, ABclonal,USA), Caspase3 ( 1:1,000 dilution, \# 19677-1-AP, Proteintech, China), rH2AX (1:1,000 dilution,\#3222535-1, Abcam), Ku80 (1:1,000 dilution, \#A5862\#, ABclonal,USA),TRF2 (1:1,000 dilution, \# 3224856-2, Abcam,USA). For the experiments under irradiation, the proteins were extracted from the irradiated cells with an adequate dose of irradiation and incubated to confluence. The Enhanced Chemiluminescence system ECL (Adanster, USA) was used to visualize the separated proteins bands and chemical dose XRS + System ( Bio-Rad, Hercules,CA,USA) and Image JProgram were respectively used to capture and analyzed (quantify) the generated images.

\section{Colony Formation Assay}

The colony formation assay was performed to evaluate the survival fraction of cells with different treatments. The stably transfected cells ( 300 cells/ $2 \mathrm{~mL} /$ well) were plated in 6 wells plate. After $24 \mathrm{~h}$ post-culture, the cells were irradiated with a single dose of 0,2 and 4 Gy using the small animal radiation research platform (SARRP, Px I X-RAD $225 \mathrm{Cx}, \mathrm{CT}$, USA, from a $204 \mathrm{Kv}$ photon beam. At 14 days post-incubation, the developed colonies were washed with PBS, fixed and sustained with crystal violet ( $1 \%$ in absolute ethanol). Cell survival was detected by counting the colonies containing $>50$ cells. The survival fraction of cells of each irradiation dose group was determined as a plating efficiency of the irradiated cell divided by that of the non-irradiated control.

\section{Cell Viability and cell proliferation assay}

Stale transfected MDA-MB-468 cells were seeded in 96 wells plates and cultured for $24 \mathrm{~h}$ at 3.103 cells $/ 100 \mu \mathrm{L} /$ well. For cells viability analysis, cells were seeded and immediately irradiated with a single dose of IR $(0,2,4,6$, and $8 \mathrm{~Gy}$ ). At $72 \mathrm{~h}$ post-incubation, $10 \mu \mathrm{L}$ of CCK- 8 was added to each well and incubated at $37^{\circ} \mathrm{C}$ for $1 \mathrm{~h}$ to $2 \mathrm{~h}$ following by their optic density (OD) reading at 450nm. For cell proliferation analysis, cells were seeded and irradiated with a single dose of IR (0 Gy and 2Gy). At an appropriated time ( $0 \mathrm{~h}, 6 \mathrm{~h}, 12 \mathrm{~h}, 24 \mathrm{~h}, 48 \mathrm{~h}$, and $72 \mathrm{~h})$ post- incubation, $10 \mu \mathrm{L}$ of CCK-8 was added to each well and incubated at $37^{\circ} \mathrm{C}$ for $1 \mathrm{~h}$ to $2 \mathrm{~h}$. The plates were read at $450 \mathrm{~nm}$ by using a 96 well plate reader. Each experiment 
was repeated three times.

Cell Cycle and Cell apoptosis analysis

Cell cycle was studied on stable transfected triple-negative breast cancer cells without and with 6Gy irradiation. At $72 \mathrm{~h}$ post-culture, the 5.105 cells plated in 6 wells plate were harvested, fixed in $70 \%$ ethanol for 30 min and stained with $500 \mu \mathrm{L}$ of propidium iodure $(\mathrm{C} 1052$, Beyotime, China) for $30 \mathrm{~min}$ at $37^{\circ} \mathrm{C}$.

Apoptosis analysis was performed by using FITCConjugated annexin V/Propidium iodide method (Annexin V-FITC/PI apoptosis Kit best bio/ China) according to the manufacturer's instructions. Samples were analyzed by flow cytometry (FACS, AriaIII, and BD, USA) and all tests were repeated three times (in Triplicate).

\section{Statistical Analysis}

Statistical analysis was performed with GraphPad Prism 5.0; the comparison of multiple groups was performed with one way ANOVA and Turkey's method. Data are expressed as mean \pm Standard Deviation (SD) and $\mathrm{P}<0,05$ was considered as an indicator of statistical significance.

\section{Results}

Down-regulation of TMPRSS4 increased the radiosensitivity of $M D A-M B-468$ cells

To explore the role of TMPRSS4 in triple negative breast cancer cells radiosensitivty, TMPRSS4 was down-regulated by transfecting TMPRSS4 silenced lentivirus vector in MDA-MB-468 breast cancer cell line which has been reported to overexpress TMPRSS4
TMPRSS4 and Triple Negative Breast Cancer Radiosensitivity

(Cheng et al.,2013; Li et al.,2017; Assani et al., 2019). Colony formation assay and CCK- 8 assay was used for performing cell viability analysis. The CCK- 8 test indicated that IR decreased the viability of the cell in a dose-dependent manner (Figure 1A) associated with the high decrease at 8 Gy in MDA-MB-468-shTMPRSS4 groups compared to MDA-MB-468-NC [(53.00\% \pm $4.6 \%$ vs $82,22 \% \pm 2.3 \%$ ); Figure $1 \mathrm{~A} ; * * * \mathrm{P}<0.001)]$. Moreover, colony formation assay showed that TMPRSS4 silencing significantly decrease colony number (reported by relative survival fraction) in irradiation dose-dependent manner (0,2 and 4Gy) with a high decrease at 4 Gy in MDA-MB-468-shTMPRSS4 groups compared to MDA-MB-468-NC (Figure 1B, ${ }^{*} * \mathrm{P}<0.001$; Figure 1C, $* \mathrm{P}<0,05)$ This result suggests that TMPRSS4 silencing improves TNBC radiosensitivity.

Effect of radiation on cell proliferation in control and TMPRSS4 down-regulated TNBC cells

To examine the synergic effect of TMPRSS4 down-regulation and 2 Gy irradiation on TNBC proliferation, CCK- 8 assay was used and MDA-MB-468 proliferation ability was determined in shTMPRSS4, 2 Gy irradiation and shTMPRSS +2 Gy IR treatment groups. Our results showed that TMPRSS4 inhibition associated with 2 Gy IR significantly reduced the MDA-MB-468 cells proliferation ability with high reduction in MDA-MB-468-shTMPRSS4 cells compared to control $[(34,52 \% \pm 2,8 \%$ vs $73,81 \% \pm 3,5 \%)$; Figure $2 \mathrm{C} ; * * * \mathrm{P}<0.001]$ than shTMPRSS $4[(44,49 \% \pm 1,8 \%$ vs $73,81 \% \pm 3,5 \%$ ); Figure $2 \mathrm{~A} ; * * * \mathrm{P}<0.001]$ and 2 Gy irradiation alone $[(54,52 \% \pm 2,8 \%$ vs $73,81 \% \pm 3,5 \%)$; Figure $2 \mathrm{~B} ; * * * \mathrm{P}<0.001]$. This result suggest that TMPRSS4
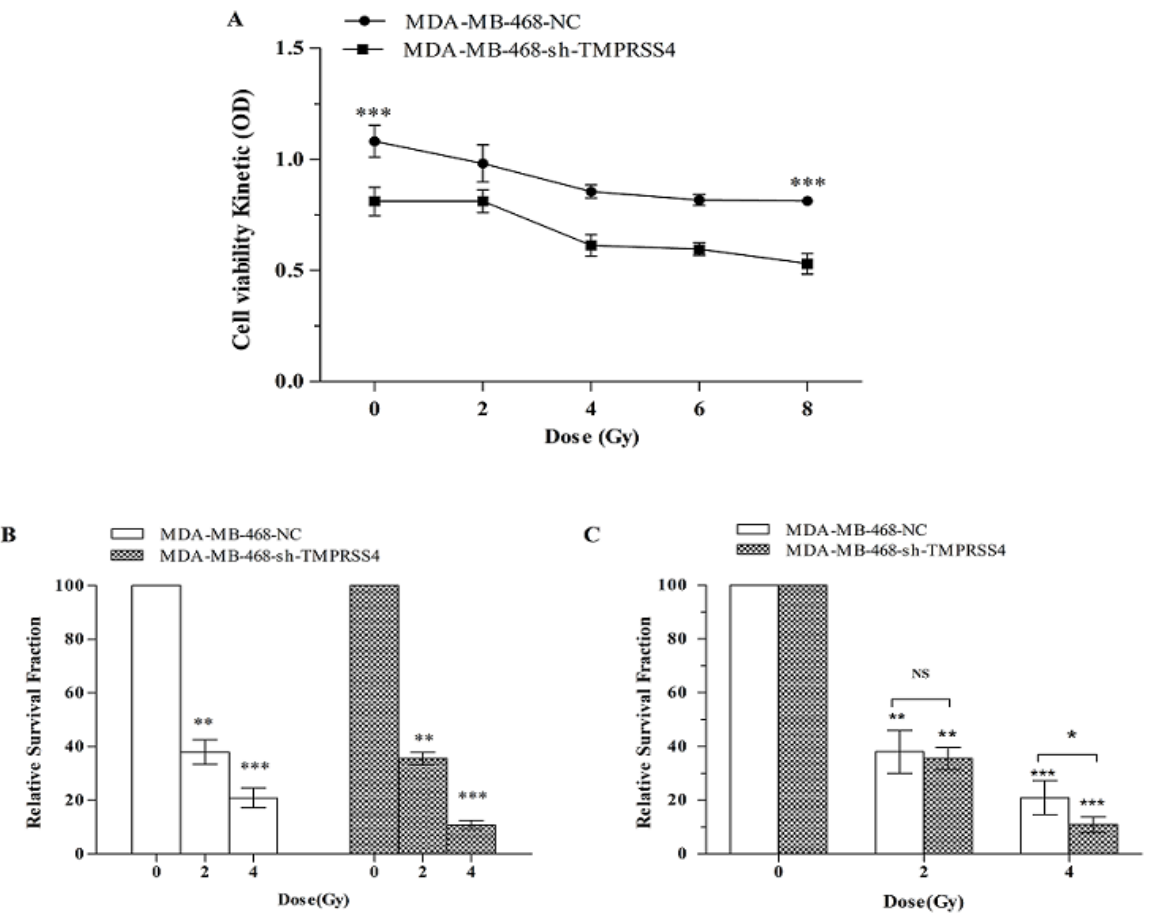

Figure 1. Down-Regulation of TMPRSS4 Expression Enhances TNBC Radiosensitivity. Stable transfected MDA-MB-468 cells were exposed to different dose of IR $(0,2,4,6,8 \mathrm{~Gy})$ followed by cell viability evaluation using CCK-8 assay (Figure 1A). The stable transfected MDA-MB-468 cells were irradiated at dose 0,2 and 4Gy following by the counting of colony development (Figure $1 \mathrm{~B}, \mathrm{C}$ ). Data represent mean \pm Standard Deviation (SD). $* \mathrm{P}<0.05 ; * * \mathrm{P}<0.01$ and $* * * \mathrm{P}<0.001$. 


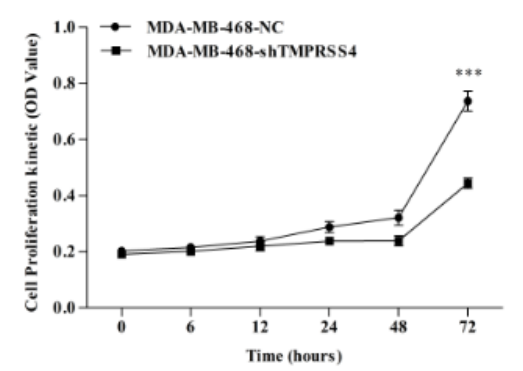

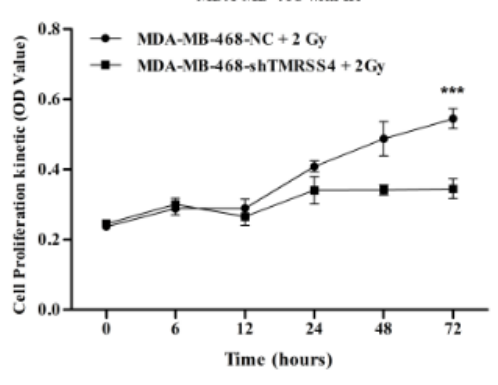

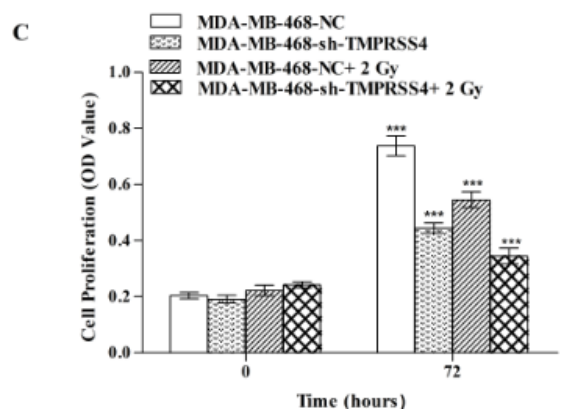

Figure 2. TMPRSS4 Silencing Enhanced TNBC Cell Proliferation Reduction Mediated by IR. The CCK-8 assay was used to detect reduction of the proliferation capacity of stable transfected MDA-MB-468 cell without IR (Figure 2 A,E) and with 2 Gy IR (Figure 2 B, C). MDA-MB-468 cell showed a remarkably cell proliferation reduction at $72 \mathrm{~h}$ post-IR in combined treatment groups than shTMPRSS4 or $2 \mathrm{~Gy}$ IR alone (Figure 2C). Values are indicated as mean $\pm \mathrm{SD}$ of three independent experiments.

down-regulation enhances cell proliferation reduction induced by $2 \mathrm{~Gy}$ irradiation Effect of radiation on cell cycle in control and TMPRSS4 down-regulated TNBC cells.

To assess the effect TMPRSS4 inhibition in radiation induced cell cycle process impairment, the stable transfected MDA-MB-468 cells and irradiated with 0 and 6 Gy IR were harvested for cell cycle analysis through flow cytometry analysis and western blot analysis of
cyclinD1 expression. Our results showed a significant increase of the rate of the cells arrested in G2/M phase in MDA-MB-468-sh-TMPRSS4 associated with 6 Gy irradiation groups $[(30,33 \% \pm 1,15 \%$ vs $19,60 \% \pm 1,21 \%)$, Figure A,C; $* * * \mathrm{P}<0.001)]$ than 6 Gy IR treatment alone [ $(28,33 \% \pm 0,57 \%$ vs $19,60 \% \pm 1,21 \%$ Figure $3 \mathrm{~A}, \mathrm{C}$; $* * * \mathrm{P}<0.001)$. Moreover, the cyclin D1 expression was significantly inhibited in TMPRSS4 silencing groups than
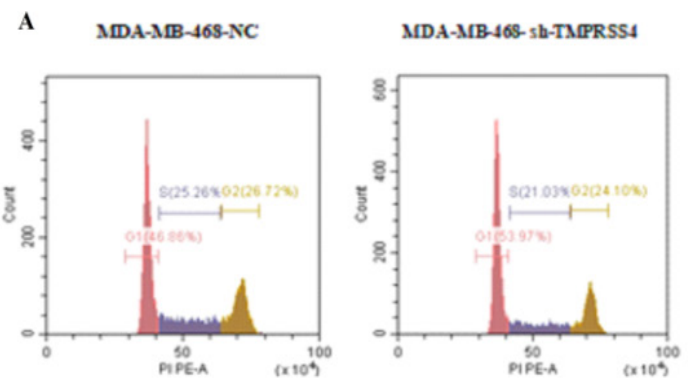

MPA-MB-468- NC+6 Gy
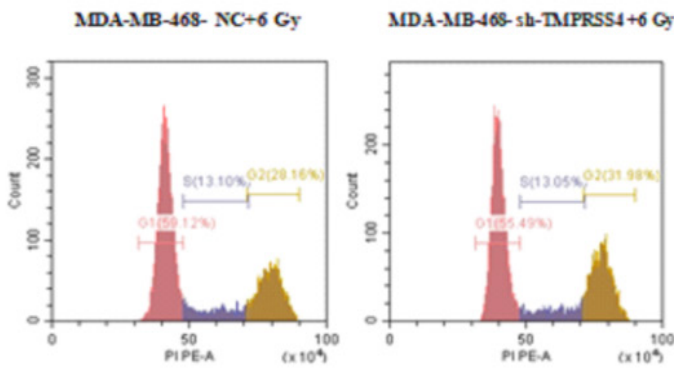

B
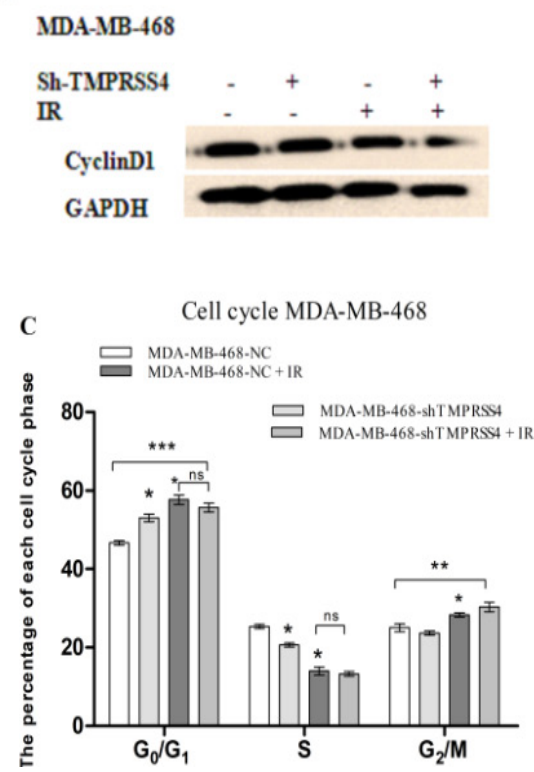

Figure 3. Down-Regulation of TMPRSS4 Expression Improved MDA-MB-468 Cell Arrested in G2/M Phase Induced by 6 Gy IR. Cell cycle analysis was performed by utilizing Flow cytometer analysis in stable transfected MDBMB-468 cells without IR (Figure 3A, B) and with 6 Gy IR (Figure C, B). Experiments were performed in triplicate $(* * * \mathrm{P}<0.001)$. Western Blot was also performed regarding the expression of CyclinD1 related to cell cycle pathway (Figure3D). 
A
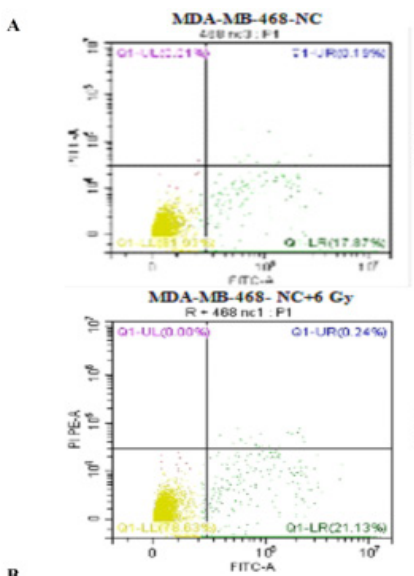
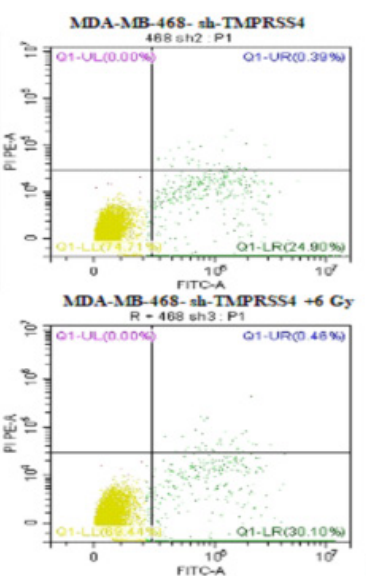
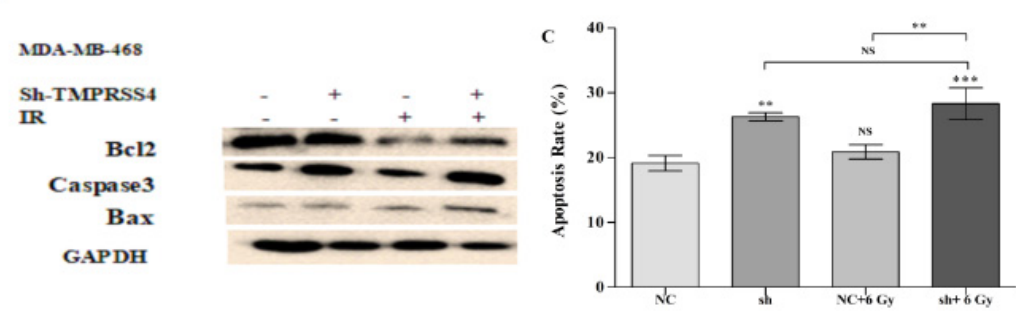

Figure 4. Inhibition of TMPRSS4 Expression Enhanced IR Induced Cell Apoptosis in MDA-MB-468 Cells. The cell apoptosis was detected via the Annexin V staining assay in stable transfected MDA-MB-468 cells without (Figure 4A, B) and with (Figure 4C, B). Values represent the mean $\pm \mathrm{SD}$ of three independent experiments $(* * * \mathrm{P}<0,001)$. Western Blot analysis of certain protein (Bax, $\mathrm{Bcl} 2$, and Caspase3) related to apoptosis pathways was also investigated in a stably transfected cell under and not 6 Gy IR (Figure 4D)

control groups after 6 Gy irradiation (Figure 3B). Our results demonstrated that TMPRSS4 silencing increased G2/M cell cycle arrested induced by IR which may due to cyclin D1 inhibition.
Effect of radiation on cell apoptosis in control and TMPRSS4 down-regulated TNBC cells

The impact of inhibition of TMPRSS4 expression on radiation induced cell apoptosis was studied by performing

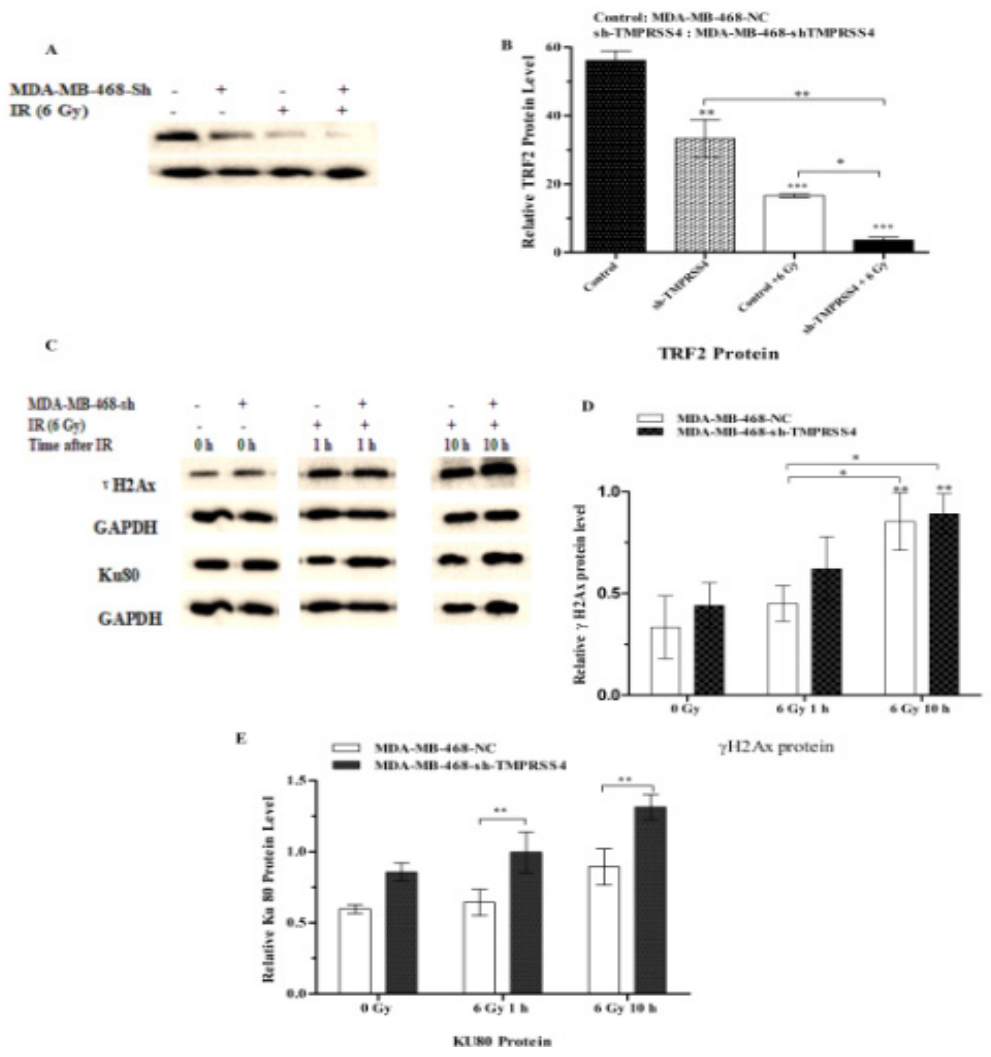

Figure 5. TMPRSS4 Silencing Improved the DNA Damage Induced by IR, Delays DNA Damage Repair. Western Blot was performed to detect in stable transfected MDA-MB-468 cell the expression level of certain proteins TRF2 and $\left(\mathrm{Ku} 80,{ }^{\mathrm{H}} 2 \mathrm{Ax}\right)$ related to DNA damage (Figure 5A,B) and DNA damage repair (Figure 5C,D,E). 
Annexin V assay and western blot assay for checking the expression of $\mathrm{Bcl}$, Bax and caspase3. The apoptotic index was significantly increased in MDA-MB-468-shTMPRSS4 groups under irradiation $[(28,33 \% \pm 2,44 \%$ vs $19,13 \%$ $\pm 1,18 \%$ ); Figure 4A,B ;***P $<0.001]$ associated with over-expression of pro-apoptotic protein (Caspase 3, Bax) and down-regulation anti-apoptotic protein $(\mathrm{Bcl})$ (Figure 4C) than in shTMPRSS4 groups [( 26,29\% $\%$ 0,62\% vs $19,13 \% \pm 1,18 \%$ ); Figure $4 \mathrm{C}, \mathrm{B} ; * * \mathrm{P}<0.01]$. Few percentage of MDA-MB-468 cells underwent apoptosis in 6 Gy irradiation treatment alone groups compared to control [( 20,89\% $\pm 1,09 \%$ vs $19,13 \% \pm 1,18 \%)$; Figure 4A,B,C ; P (NS)]. This data demonstrate that TMPRSS4 inhibition improved 6 Gy IR induced MDA-MB-468 cells apoptosis.

Down-regulation of TMPRSS4 enhances DNA damage and delays DNA damage repair induced by IR in TNBC DNA damage is the most important target of radiation induced cell damage and TRF 2 degradation is reported to illustrated the DNA damage response (Kwon et al., 2013; Zhang et al., 2006). To investigate whether TMPRSS4 silencing resulted in a change in IR- induced TNBC DNA damage, TRF2 expression level using western blot was evaluated in MDA-MB-468-shTMPRSS4 and its control with and without 6 Gy IR. TRF2 levels is significantly decreased when radiation was combined with TMPRSS4 silencing than radiation alone or TMPRSS4 silencing alone (Figure 5A,B; ;** $\mathrm{P}<0.001$ ). Ku 80 and ${ }^{8} H 2 A x$ expression were investigated at $1 \mathrm{~h}$ and $10 \mathrm{~h}$ post- IR for DNA damage repair analysis. The expression of $K u 80$ and ${ }^{r} H 2 A x$ is continuously increased at $1 \mathrm{~h}$ post and $10 \mathrm{~h}$ post- IR with the high increase in MDA-MB-468-shTMPRSS4 groups (Figure 5.A, B, C); suggested that TMPRSS4 silencing increased the DNA damage induced by IR and delayed the DNA damage repair. Relative gray value $=$ Gtarget protein $/$ GGAPDH (G: gray value) $* \mathrm{P}<0.05$. The experiments were repeated three times independently.

\section{Discussion}

Radiotherapy is one of the most effective breast cancer treatment strategies which significantly improves the therapeutic outcome and survival of breast cancer patients (Moran et al., 2015; Bartelink et al., 2001; Fisher et al., 1995; Cun et al., 2013). Resistance to radiation represents one of the important challenges for breast cancer radiotherapy effectiveness including triple negative breast cancer (Ko et al., 2018; Qi et al.,2017; He et al., 2018) which represent the most radio-resistant breast cancer (He et al., 2018; Kyndi et al., 2008). In the present study, found that TMPRSS4 inhibition increased TNBC cell radio-sensitivity (Figure 1A, B, C) and improved the cell proliferation reduction mediated by IR (Figure 2C). Moreover, the cell cycle is an important step in cancer development (William et al., 2012; Senderowiez et al., 2002) and cancer treatment such as radiotherapy (Assani et al., 2019; Pawlik et al., 2004). Additionally, cyclin D1 is one of the proteins related to cell cycle pathway (Casimiro et al., 2012) and radiotherapy (Shimura et al., 2011). Our results indicated that TMPRSS4 silencing increased the cell arrested in G2/M phase mediated by irradiation associated with cyclinD1 expression inhibition. This suggested that TMPRSS4 silencing enhanced the TNBC cell radio-sensitivity via cell cycle arrested in $\mathrm{G} 2 / \mathrm{M}$ phase (Figure 3B,C). It has been reported that TMPRSS4 silencing increased cell arrested in the G2/M phase (Assani et al., 2019), ionizing radiation also caused cell arrested in the G2/M phase (Zhao et al., 2019; Dillon et al., 2014; Yan et al., 2007; Luo et al.,2018), cyclin D1 is induced in the G2 phase under Ras pathways control in rapidly proliferation cell (Sherr et al., 2002), cyclinD1 down-regulation sensitize cell to irradiation $(\mathrm{Su}$ et al., 2015; Marampon et al., 2016) and cells are more radiosensitive at G2/M phase (Palik et al., 2004). This suggested that TMPRSS4 down-regulation improved TNBC cell radio-sensitivity may be due to cyclin D1 inhibition and accumulation of $\mathrm{G} 2 / \mathrm{M}$ arrested cells. However, further studies are needs to more investigate the implication of the Ras pathway in relationship between cyclin D1, G2/M, and radio-sensitivity of TNBC cells.

Otherwise, cell apoptosis is one of the keys even in cell life (David et al., 1999) and could be a target of cancer treatment strategy or cancer treatment adjuvant such as radiotherapy (Du et al., 2006; Liu et al., 2011).

The pro-apoptotic protein (Bax and Caspase 3 ) and the anti-apoptotic protein $(\mathrm{BCl})$ are the kinds of the proteins related to cell apoptosis pathway and implicated in cancer radiotherapy (Mackey et al., 1998; Dong et al., 2016; Kaliberov et al., 2002). In the present study, we found that TMPRSS4 down-regulation improved the apoptosis effect of ionizing radiation on triple negative breast cancer (Figure 4A, B) associated with inhibition of expression of $\mathrm{Bcl} 2$ and increased of Bax and Caspase 3 (Figure 4C). Our previous study reported that TMPRSS4 expression inhibition induced cell apoptosis following by Bax and Bcl2 expression modulation (Assani et al., 2019) and numerous studies reported that IR promoted cells apoptosis (Assani et al., 2019). Moreover, inhibition of $\mathrm{Bcl} 2$ and up-regulation of Bax and caspase 3 expression were also reported to be associated with increased cancer radiosensitivity through an increase of cancer cell apoptotic index (Jiang et al., 2016). Nevertheless, the low rate of MDA-MB-468 cells underwent to apoptosis in irradiation alone groups may be due to the lack of estrogen receptor (ER) in MDA-MB-468 cells as it has been that ER transfection enhanced radiosensitivity of TNBC cells via additional apoptosis induction (Chen et al., 2017). Based on these data, TMPRSS4 silencing enhanced TNBC radiosensitivity may be due to increase of apoptotic index following by $\mathrm{Bcl} 2$, Bax and caspase 3 expression impairment The DNA damage is one of the important effects of ionizing radiation on the cancer cells and the inability of cancer cells to repair that DNA damage characterizes the radiotherapy effectiveness (Alizadeh et al., 2015; Mladenov et al., 2013). TRF2 and (Ku80, ${ }^{8} \mathrm{H} 2 \mathrm{AX}$ ) are proteins related to DNA damage (Sharma et al., 2012; Wang et al., 2014) and DNA damage repair. We found that TMPRSS4 silencing enhanced the DNA damage induced by through TRF2 inhibition (Figure 5A, B). Subsequently, the complete repair of DNA damage would occur within $30 \mathrm{~min}$ to $5 \mathrm{~h}$ with the loss of almost DNA damage protein within $7 \mathrm{~h}-8 \mathrm{~h}$ post IR (Löbrich et 
al., 2010; Nazarov et al., 2003; Solovjeva et al., 2009; Svetlova et al., 2007). In the present study, we found that TMPRSS4 inhibition delayed DNA damage repair in TNBC cell lines (Figure 5C, D, E) by continuously increasing $\mathrm{Ku} 80$ and ${ }^{r} \mathrm{H} 2 \mathrm{AX}$ expression at $1 \mathrm{~h}$ and at $10 \mathrm{~h}$ post-IR. TMPRSS4 down-regulation increased TNBC cell radiosensitivity may due to improve of DNA damage and delay of DNA damage repair. However, the mechanism by which TMPRSS4 down-regulation enhanced IR induced DNA damage and delays DNA damage repair remains more investigate. In conclusion, our results indicated that down-regulation of TMPRSS4 improved triple negative breast cancer radiosensitivity via cells arrested in $\mathrm{G} 2 / \mathrm{M}$ phase and increase of cells apoptosis ability. This provide the new information for novel combination of therapies synergic using sh-TMPRSS4 with radiation in patient with TNBC.

\section{Acknowledgments}

The authors thank members of our laboratories for helping to process the works.

\section{Funding}

China Scholarship Council (Serial number: 351569)

\section{Authors'contributions}

Y.F.Z, F.X.Z, and G.A designed the present study. G.A, Y.D.X, and Y.X.Y performed experiment Work. A.Y and J.S were involved in experiment work execution. G.A processed the experimental data, performed the analysis and drafted the manuscript.

\section{Benin contributors informations}

Julien Segbo : julien.segbo@epac.uac.bj

Akadiri Yessoufou: akadiri.yessoufou@gmail.com

\section{Competing interests}

The authors declare that they have no competing interests.

\section{References}

Abramson VG, Lehmann BD, Ballinger TJ, Pietenpol JA(2015), Subtyping of triple-negative breast cancer: implications for therapy. Cancer, 121, 8-16.

Agrawal LS, Mayer IA (2014). Platinum agents in the treatment of early-stage triple-negative breast cancer: is it time to change practice?. Clin Adv Hematol Oncol, 12, 654-8.

Alizadeh E, Orlando TM, Sanche L (2015). Biomolecular damage induced by ionizing radiation: the direct and indirect effects of low-energy electrons on DNA. Annu Rev Phys Chem, 66, 379-98.

Assani G, Yessoufou A, Xiong Y, et al (2019). Role of TMPRSS4 Modulation in Breast Cancer Cell Proliferation. Asian Pac $J$ Cancer Prev, 20, 1849-56.

Assani G, Zhou Y (2019). Effect of modulation of epithelial-mesenchymal transition regulators Snaill and Snail2 on cancer cell radiosensitivity by targeting of the cell cycle, cell apoptosis and cell migration/invasion. Oncol Lett, 17, 23-30.

Augoff K, McCue B, Plow EF, Sossey-Alaoui K (2012). miR-31 and its host gene lncRNA LOC554202 are regulated
TMPRSS4 and Triple Negative Breast Cancer Radiosensitivity

by promoter hypermethylation in triple-negative breast cancer. Mol Cancer, 11, 5.

Bartelink H, Horiot JC, Poortmans P, et al (2001). Recurrence rates after treatment of breast cancer with standard radiotherapy with or without additional radiation. $N$ Engl $J$ Med, 345, 1378-87.

Bray F, Ferlay J, Soerjomataram I, et al (2018). Global cancer statistics 2018: GLOBOCAN estimates of incidence and mortality worldwide for 36 cancers in 185 countries. $C A$ Cancer J Clin, 68, 394-424.

Casimiro MC, Crosariol M, Loro E, Li Z, Pestell RG (2012). Cyclins and cell cycle control in cancer and disease. Genes Cancer, 3, 649-57.

Chen W, Zheng R, Baade PD, et al,(2016). Cancer statistics in China, 2015. CA Cancer J Clin, 66, 115-32.

Cheng D, Kong H, Li Y (2013). TMPRSS4 as a poor prognostic factor for triple-negative breast cancer. Int J Mol Sci, 14, 14659-68.

Chen X, Ma N, Zhou Z, et al (2017). Estrogen receptor mediates the radiosensitivity of triple-negative breast cancer cells. Med Sci Monit, 23, 2674-83.

Chikaishi Y, Uramoto H, Koyanagi Y, et al (2016). TMPRSS4 expression as a marker of recurrence in patients with lung cancer. Anticancer Res, 36, 121-7.

Cun Y, Dai N, Xiong C, et al (2013). Silencing of APE1 enhances the sensitivity of human hepatocellular carcinoma cells to radiotherapy in vitro and in a xenograft model. PLoS One, 8, e55313

David LV, Stanley JK (1999). Cell death in development review. Cell, 96, 245-54.

Diaz-Moralli S, Tarrado-Castellarnau M, Miranda A, Cascante M (2013). Targeting cell cycle regulation in cancer therapy. Pharmacol Ther, 138, 255-71.

Dillon MT, Good JS, Harrington KJ (2014). Selective targeting of the G2/M cell cycle checkpoint to improve the therapeutic index of radiotherapy. Clin Oncol (R Coll Radiol), 26, 257-65.

Dong Y, Zheng Y, Xiao J, Zhu C, Zhao M (2016). Regulatory effect of Bcl-2 in ultraviolet radiation-induced apoptosis of the mouse crystalline lens. Exp Ther Med, 11, 973-7.

Fan X, Liang Y, Liu Y, et al (2018). The upregulation of TMPRSS4, partly ascribed to the downregulation of miR $125 \mathrm{a} 5 \mathrm{p}$, promotes the growth of human lung adenocarcinoma via the NF $\kappa$ B signaling pathway. Int J Oncol, 53, 148-58.

Fisher B, Anderson S, Redmond CK, et al (1995). Reanalysis and results after 12 years of follow-up in a randomized clinical trial comparing total mastectomy with lumpectomy with or without irradiation in the treatment of breast cancer. $N$ Engl J Med, 333, 1456-61.

Foulkes WD, Smith IE, Reis-Filho JS (2010). Triple-negative breast cancer. $N$ Engl J Med, 363, 1938-48.

Ilgun S, Sarsnov D, Erdogan Z et al (2016). Receptor discordance rate and its effects on survival in primary and recurrent breast cancer patients. $J$ BUON, 21, 1425-32.

He MY, Rancoule C, Rehailia-Blanchard A, et al (2018). Radiotherapy in triple-negative breast cancer: Current situation and upcoming strategies. Crit Rev Oncol Hematol, 131, 96-101.

Huang A, Zhou H, Zhao H, et al (2014). TMPRSS4 correlates with colorectal cancer pathological stage and regulates cell proliferation and self-renewal ability. Cancer Biol Ther, 15, 297-304.

Jiang F, Zhou L, Wei C, Zhao W, Yu D (2016). Slug inhibition increases radiosensitivity of oral squamous cell carcinoma cells by upregulating PUMA. Int J Oncol, 49, 709-19.

Jung H, Lee KP, Park SJ, et al ( 2008). TMPRSS4 promotes invasion, migration and metastasis of human tumor cells by 
facilitating an epithelial-mesenchymal transition. Oncogene, 27, 2635-47.

Kadam CY, Abhang SA (2016). Apoptosis markers in breast cancer therapy. Adv Clin Chem, 74, 143-93.

Kaliberov S, Buchsbaum DJ, Gillespie GY, et al (2002). Adenovirus mediated transfer of Bax driven by the vascular endothelial growth factor promoter induces apoptosis in lung cancer cells. Mol Ther, 6, 190-8.

Kindts I, Buelens P, Laenen A, et al (2017). Omitting radiation therapy in women with triple-negative breast cancer leads to worse breast cancer-specific survival. Breast J, 32, 18-25.

Kim S, Kang HY, Nam EH, et al (2010). TMPRSS4 induces invasion and epithelial-mesenchymal transition through upregulation of integrin alpha5 and its signaling pathways. Carcinogenesis, 31, 597-606.

Ko YS, Jin H, Lee JS, et al (2018). Radioresistant breast cancer cells exhibit increased resistance to chemotherapy and enhanced invasive properties due to cancer stem cells. Oncol Rep, 40, 3752-62.

Kyndi M, Sørensen FB, Knudsen H, et al (2008). Estrogen receptor, progesterone receptor, HER-2, and response to post-mastectomy radiotherapy in high-risk breast cancer: the Danish breast cancer cooperative group. J Clin Oncol, 26, 1419-26.

Lee A, Djamgoz MBA (2018). Triple-negative breast cancer: Emerging therapeutic modalities and novel combination therapies. Cancer Treat Rev, 62, 110-22.

Lee Y, Ko D, Min HJ, et al (2016). TMPRSS4 induces invasion and proliferation of prostate cancer cells through induction of Slug and cyclin D1. Oncotarget, 7, 50315-32.

Lehmann BD, Bauer JA, Chen X, et al (2011). Identification of human triple-negative breast cancer subtypes and preclinical models for selection of targeted therapies. $J$ Clin Invest, 121, 2750-67

Liang B, Wu M, Bu Y, Zhao A, Xie F (2013). Prognostic value of TMPRSS4 expression in patients with breast cancer. Med Oncol, 30, 497.

Li T, Zeng ZC, Wang L, et al (2011). Radiation enhances long-term metastasis potential of residual hepatocellular carcinoma in nude mice through TMPRSS4-induced epithelial-mesenchymal transition. Cancer Gene Ther, 18, 617-26.

Li XM, Liu WL, Chen X, et al (2017). Overexpression of TMPRSS4 promotes tumor proliferation and aggressiveness in breast cancer. Int J Mol Med, 39, 927-35.

Löbrich M, Shibata A, Beucher A, et al (2010). gammaH2AX foci analysis for monitoring DNA double-strand break repair: Strengths, limitations and optimization. Cell Cycle, 9, 662-9.

Lukoseviciene V, Tikuisis R, Dulskas A, Miliauskas P, Ostapenko V (2018). Surgery for triple-negative breast cancer does the type of anesthesia have an influence on oxidative stress, inflammation, molecular regulators and outcome of disease? J BUON, 23, 290-5.

Luo Y, Chen X, Luo L, Zhang Q, et al (2018). [6]-Gingerol enhances the radiosensitivity of gastric cancer via G2/M phase arrest and apoptosis induction. Oncol Rep, 39, 2252-60.

Mackey TJ, Borkowski A, Amin P, Jacobs SC, Kyprianou N (1998). bcl-2/bax ratio as a predictive marker for therapeutic response to radiotherapy in patients with prostate cancer. Urology, 52, 1085-90.

Marampon F, Gravina G, Ju X, et al (2016). Cyclin D1 silencing suppresses tumorigenicity, impairs DNA double strand break repair and thus radiosensitizes androgen-independent prostate cancer cells to DNA damage. Oncotarget, 7, 5383-400.

Meyn RE (1997). Apoptosis and response to radiation: implications for radiation therapy. Oncology (Williston Park), 11, 349-56.

Millikan RC, Newman B, Tse CK, et al (2008). Epidemiology of basal-like breast cancer. Breast Cancer Res Treat, 109, 123-39.

Mladenov E, Magin S, Soni A, et al (2013). DNA double-strand break repair as determinant of cellular radiosensitivity to killing and target in radiation therapy. Front Oncol, 10, 113.

Moran MS (2015). Radiation therapy in the locoregional treatment of triple-negative breast cancer. Lancet Oncol, 16, 113-22.

Nazarov IB, Smirnova AN, Krutilina RI, et al (2003). Dephosphorylation of histone gamma-H2AX during repair of DNA double-strand breaks in mammalian cells and its inhibition by calyculin A. Radiat Res, 160, 309-17.

Pawlik TM, Keyomarsi K (2014). Role of cell cycle in mediating sensitivity to radiotherapy. Int J Radiat Oncol Biol Phys, 59, 928-42.

Pistritto G, Trisciuoglio D, Ceci C, Garufi A, D’Orazi G (2016). Apoptosis as anticancer mechanism: function and dysfunction of its modulators and targeted therapeutic strategies. Aging (Albany NY), 8, 603-19.

Qi XS, Pajonk F, McCloskey S, et al (2017). Radioresistance of the breast tumor is highly correlated to its level of cancer stem cell and its clinical implication for breast irradiation. Radiother Oncol, 124, 455-61.

Senderowicz AM (2002). The cell cycle as a target for cancer therapy: basic and clinical findings with the small molecule inhibitors flavopiridol and UCN-01. Oncologist, 7, 12-9.

Sharma A, Singh K, Almasan A (2012). Histone H2AX phosphorylation: a marker for DNA damage. Methods $\mathrm{Mol}$ Biol, 920, 613-26.

Sherr CJ (2002). D1 in G2. Cell Cycle, 1, 36-8.

Shimura T, Kakuda S, Ochiai Y, et al (2011). Targeting the AKT/ GSK3 $\beta /$ cyclin D1/Cdk4 survival signaling pathway for eradication of tumor radioresistance acquired by fractionated radiotherapy. Int J Radiat Oncol Biol Phys, 1, 540-8.

Solovjeva LV, Pleskach NM, Firsanov DV, et al (2009). Forskolin decreases phosphorylation of histone $\mathrm{H} 2 \mathrm{AX}$ in human cells induced by ionizing radiation. Radiat Res, 171, 419-24.

Su H, Jin X, Shen L, et al (2016). Inhibition of cyclin D1 enhances sensitivity to radiotherapy and reverses epithelial to mesenchymal transition for esophageal cancer cells. Tumour Biol, 7, 5355-63.

Svetlova M, Solovjeva L, Nishi K, et al (2007). Elimination of radiation-induced gamma-H2AX foci in mammalian nucleus can occur by histone exchange. Biochem Biophys Res Commun, 358, 650-4.

Tian Q, Wang Y, Guo H et al (2017). Recent perspectives of management of breast cancer metastasis; an update. JBUON, 22, 295-300.

Torre LA, Bray F, Siegel RL, et al (2015). Global cancer statistics, 2012. CA Cancer J Clin, 65, 87-108.

Torre LA, Siegel RL, Ward EM, Jemal A (2016). Global cancer incidence and mortality rates and trends-an update. Cancer Epidemiol Biomarkers Prev, 25, 16-27.

Wang FZ, Fei HR, Cui YJ, et al (2014). The checkpoint 1 kinase inhibitor LY2603618 induces cell cycle arrest, DNA damage response and autophagy in cancer cells. Apoptosis, 19, 1389-98.

Wen Y, Dai G, Wang L, Fu K, Zuo S (2019). Silencing of XRCC4 increases radiosensitivity of triple-negative breast cancer cells. Biosci Rep, 39.

Williams GH, Stoeber K (2012). The cell cycle and cancer. $J$ Pathol, 226, 352-64.

Wong RS (2011). Apoptosis in cancer: from pathogenesis to treatment. J Exp Clin Cancer Res, 30, 87. 
Wu XY, Zhang L, Zhang KM, et al (2014). Clinical implication of TMPRSS4 expression in human gallbladder cancer. Tumour Biol, 35, 5481-6.

Yang M, Zeng C, Li P, et al (2019). Impact of CXCR4 and CXCR7 knockout by CRISPR/Cas9 on the function of triple-negative breast cancer cells. Onco Targets Ther, 12, 3849-58.

Yan Y, Black CP, Cowan KH (2007). Irradiation-induced G2/M checkpoint response requires ERK1/2 activation. Oncogene, 26, 4689-98.

Zhang P, Furukawa K, Opresko PL, et al (2006).TRF2 dysfunction elicits DNA damage responses associated with senescence in proliferating neural cells and differentiation of neurons. J Neurochem, 97, 567-81.

Zhao H, Zhuang Y, Li R, et al (2019). Effects of different doses of $\mathrm{X}$-ray irradiation on cell apoptosis, cell cycle, DNA damage repair and glycolysis in HeLa cells. Oncol Lett, 17, 42-54.

\section{(c) (1) (3)}

This work is licensed under a Creative Commons AttributionNon Commercial 4.0 International License. 